\title{
AN ESSAY ON STELLAR OSCILLATIONS AND EVOLUTION***
}

\author{
(Invited Review)
}

ICKO IBEN, Jr.

Dept. of Astronomy, University of Illinois, Urbana, Illinois, U.S.A.

\begin{abstract}
It is cautioned that solar models adjusted in such a way as to achieve a match between theoretical solar oscillation characteristics and observed ones may produce neutrino fluxes inconsistent with the observations and that this is likely to be explicable as a deficiency in modeling that portion of the envelope which is most strongly affected by uncertainties in the treatment of convection. Then follows a summary of how the results of pulsation theory and of stellar evolution theory have been used together to learn about the structure and evolution of RR Lyrae stars, classical Cepheids, and high luminosity AGB stars.
\end{abstract}

\section{Introduction}

I have been assigned the task of showing how observations of stellar oscillations may be used to help solve problems in stellar evolution. The store of experience on stellar 'seismology' from which I will draw is restricted to large amplitude pulsators which lie in the classical instability strip (outside of which the Sun finds itself). For these pulsators, the theoretical apparatus necessary to use the observations to learn something about global properties is in most instances far less sophisticated than is required for interpreting solar oscillations. Usually only one mode is excited at large amplitude, and this one mode is radial. A beautiful review of the whole subject is given by Cox (1974).

Before beginning the main story, I will make several observations concerning attempts to deduce information about the solar interior from comparison between the mode structure of solar models and observed solar oscillatory characteristics.

\section{A Cautionary Note Concerning Interpretations of Solar Structure Based on Observed Oscillatory Properies of the Sun}

The choice of the Crimea for this conference is most fitting since the evidence for a coherent solar oscillation at a period of about $160 \mathrm{~min}$ was first discovered here (Severny et al., 1976). I recall reading the discovery paper and being very skeptical about the alleged demonstration of periodic motion. I was even more skeptical about the interpretation of the reported period as an indication that the Sun's effective polytropic index is quite different from 3 . As reported at this meeting, the quality of the data both

\footnotetext{
* Proceedings of the 66th IAU Colloquium: Problems in Solar and Stellar Oscillations, held at the Crimean Astrophysical Observatory, U.S.S.R., 1-5 September, 1981.

** Supported in part by the National Science Foundation Grant AST 81-15325.
} 
from the Crimea and from other stations around the world has improved to such an extent that it is difficult to remain skeptical about the existence of the 160 min oscillation. However, the interpretations of the period in terms of oscillatory characteristics of models of solar structure have not yet reached the same level of believability.

To dramatize the danger of taking too seriously inferences about solar structure based on comparison between observed oscillatory characteristics and results of a pulsational analysis of solar models, adopt the absurd assumption that the period, radius, and surface gravity of the Sun are related in the same way as in a pendulum for which $P \simeq 2 \pi \sqrt{l / g}$. Here $P$ is the period, $l$ is the length, and $g$ is the gravitational acceleration. Setting $l=R_{\odot}$ and $g=G M_{\odot} / R_{\odot}^{2}$, where $R_{\odot}$ and $M_{\odot}$ are the solar radius and mass, respectively, one has

$$
P \cong 10000 \mathrm{~s}=167 \mathrm{~min} .
$$

On comparison with the observed 160 min period of the Sun, one might infer that the Sun is a pendulum!

As another (slightly more serious) illustration of the dangers of interpretation, consider the oscillations of periods between about 5 and about 60 min that have been variously reported in the literature. Evidence for an oscillation at a period of about $40 \mathrm{~min}$ has in the past been published by at least four groups. Standard solar model calculations demonstrate that the period of the first solar radial overtone is about $40 \mathrm{~min}$ and is essentially independent of model details. One might therefore be tempted to infer that theory and observation have together revealed that the first radial overtone is excited in the Sun. However, at this conference, several of the authors have stated in private that their published 'observational' identifications are spurious. Several other period identifications have undergone evolutionary changes in the literature. Thus, the confidence one might have felt at relating a model-independent result to an authorindependent, published observational result has in this case shown itself to be misplaced; confidence gives way to severe skepticism. The danger illustrated by this parable is that agreement between 'observation' and a theoretical interpretation does not always mean that either the theoretical interpretation or the observation is correct.

To pursue this theme, suppose that someone were to report the detection of a long period oscillation with a period in the neighborhood of $60 \mathrm{~min}$. A stellar astronomer acquainted with classical Cepheids and RR Lyrae stars might well jump to the conclusion that the period of the fundamental radial mode of the Sun had been detected. He might well construct a series of solar models as a function of uncertain parameters in an effort to use the 'observed' period as a constraint on these parameters. In Table I (Iben and Mahaffy, 1976) is the possible outcome of such an exercise. In this table, $Z$ and $X$ are the abundances by mass of the heavy elements and of hydrogen, respectively, in the initial Sun; $T_{c}$ and $\rho_{c}$ are the current central temperature and density in units of $10^{6} \mathrm{~K}$ and $\mathrm{g} \mathrm{cm}^{-3}$, respectively, and $N_{v}$ is the solar neutrino flux at the Earth in SNU's. The mixing length to pressure scale height ratio is given by $l / H ; \Delta R$ and $\Delta M$ are the radial thickness and mass of the convective envelope, both in solar units; and $T_{\mathrm{BASE}}$ is the temperature at the base of the convective envelope in units of $10^{6} \mathrm{~K}$. Finally, $P_{0}$ 
is the period in minutes of the fundamental radial mode. In all cases the solar model is of age $4.6 \times 10^{9} \mathrm{yr}$ and its luminosity and radius are the current solar values.

It is simple to understand qualitatively the trends in Table I. The larger $Z$, the larger is the interior opacity, and therefore the larger must be central temperature be in order to permit the outward diffusion of a given solar flux. Larger central temperatures and densities are accomplished by reducing the number of particles per gram - this means a smaller abundance of hydrogen. Higher interior temperatures mean a larger neutrino flux.

TABLE I

Dependence of several solar model characteristics on composition choice

\begin{tabular}{llllll}
\hline$Z$ & $X$ & $P_{0}$ & $T_{c}$ & $\rho_{c}$ & $N_{v}$ \\
\hline 0.01 & 0.82 & 56 & 14.5 & 146 & 4.4 \\
0.02 & 0.74 & 62 & 15.2 & 165 & 10.3 \\
0.03 & 0.67 & 66 & 15.9 & 192 & 24.7 \\
\hline
\end{tabular}

\begin{tabular}{lllllll}
\hline$Z$ & $X$ & $P_{0}$ & $l / H$ & $\Delta R$ & $\Delta M$ & $T_{\text {BASE }}$ \\
\hline 0.01 & 0.82 & 56 & 0.69 & 0.16 & $1.3 \times 10^{-3}$ & 0.91 \\
0.02 & 0.74 & 62 & 1.01 & 0.23 & $1.0 \times 10^{-2}$ & 1.6 \\
0.03 & 0.67 & 66 & 1.26 & 0.32 & $4.1 \times 10^{-2}$ & 2.7 \\
\hline
\end{tabular}

For fixed $l / H$, increasing $Z$ causes the model solar radius to increase (thanks to an increase in opacity). In order to maintain the model radius fixed at the solar value, an increase in $Z$ must be compensated for by an increase in $l / H$, thereby increasing the efficiency of convection. More efficient convection in the solar envelope means a shallower temperature gradient there and a deeper and more massive convective envelope. Finally, since it is proportional to $T^{1 / 2}$, sound velocity increases inward through the envelope more slowly for larger $Z$. Since most of the time required by a sound wave to travel from center to surface is spent in the outer envelope where velocities are considerably smaller than in the deep interior, the larger $Z$, the longer is the period of the fundamental mode.

Conventional wisdom has it that $Z_{\odot} \simeq 0.015$, implying that $N_{v} \simeq 6 \mathrm{SNU}, T_{\text {BAS- }}$. $\mathrm{E} \simeq 1.25 \times 10^{6} \mathrm{~K}$, and $P_{0} \simeq 59 \mathrm{~min}$. If neutrinos have mass, then theory has it that the electron neutrino is just one of two or three states of a more universal neutrino and that, therefore, the actual counting rate should be $N_{v} /(2-3) \simeq 3-2$ SNU. This is reasonably close to the most recent experimental estimate of about 2 SNU (Davis, 1979).

Note, however, that $T_{\mathrm{BASE}}$ is too small for the element $\mathrm{Li}$ to burn within the solar lifetime, making it difficult to understand why the abundance of $\mathrm{Li}$ is so small at the stellar surface (compared to abundances 100 times larger in young stars). Within the framework of the simple mixing length theory, the only way to solve the Li problem is to assume a larger than conventional value of $Z$ (by about a factor of 2 ) and a similarly 
larger value of $l / H$. However, the associated value of $X$ is so much larger than found in the interstellar gas (or even in conventional theoretical models of the Big Bang) that it is unacceptable, and the derived value of $N_{v}$ can be reconciled with experiment only by, say, adopting the assumption of a 10 or 12 component neutrino; but this even the most adventuresome of the theoretical physicists would be reluctant to do.

Suppose, however, that a reliable solar oscillation experiment actually gave $P_{\mathrm{o}} \simeq 66 \mathrm{~min}$ ? One should actually not find this result either surprising or alarming. It would certainly be a mistake to infer that the 'observed' period demonstrates an error in at least one of: (1) estimates of $Z$; (2) the opacity associated with $Z$; (3) the solar neutrino experiment; (4) elementary particle theory. Although none of these possibilities for error is excluded, it is much more likely that the efficiency of envelope convection given by the mixing length recipe is totally in error. The correct inference would be that both $P_{0}$ and $T_{\mathrm{BASE}}$ reflect properties of the outer convective zone and that, as we have all known from the beginning, the mixing length recipe for convection is an utter failure when it comes to describing locally the actual physics of energy flow in an outer convective region. Appropriate action woul be to see if models using more sophisticated treatments of convection could produce the observed $P_{0}$ and $N_{v}$ simultaneously.

It is sobering to realize that it is the uncertainty in our treatment of convection that prevents us from estimating via pulsation theory and an observed period and radius the mass of a Main Sequence star with a deep convective envelope. If it were not for this uncertainty, one might expect a relationship of the form

$$
M=P^{2} R^{-3} f(R),
$$

where $f$ is weakly dependent on the radius $R$, to hold. From Table $\mathrm{I}$, one sees that an uncertainty of at least $20 \%$ in $P_{0}$ is associated with the uncertainty in the treatment of convection. What this means is that, even if $P_{0}$ were known, there is a $40 \%$ uncertainty in the inferred stellar mass!

\section{Classical Large-Amplitude Pulsators (Now for the Main Story)}

In the Hertzsprung-Russell diagram one may draw a line along which hydrogen at the stellar photosphere is $\frac{1}{2}$ ionized and $\frac{1}{2}$ neutral; due to the high opacity in the zone of partially ionized hydrogen, convection extends inward from somewhat above the photosphere. With increasing luminosity, this line slopes gently toward lower surface temperatures (from $\log T_{e} \simeq 3.9$ at $\log L \simeq 1$ to $\log T_{e} \simeq 3.7$ at $\log L \simeq 5$ ). As one moves to the right of the line, the base of the convective envelope extends ever deeper, reaching and extending beyond the region of partial helium ionization. As one moves to the left of the line, the region of partial hydrogen ionization moves deeper and deeper below the photosphere; and the base of the surface convective zone recedes outward. Eventually, extensive envelope convection disappears and convection is restricted to small portions of the deep hydrogen and helium ionization layers. A star within a narrow strip parallel to and slightly to the left of the line will oscillate at large amplitude in either the 
fundamental or first overtone radial mode. That it will oscillate is known from the observations. That it oscillates in one of two radial modes (in most cases) is known from a comparison between results of radial pulsation theory and observation. How a star gets into the instability strip is known from stellar evolution theory. What is responsible for the oscillation is known from pulsation theory (driving in the helium and hydrogen ionization zones). The right hand limit to the instability strip is presumably due to the development of a deep convective envelope which damps out the instability mechanism in the ionization zones. The left hand limit is due to the positioning of the driving zones so far below the stellar surface that they cannot 'lift' the layers above them.

In practice, the interplay between the observations, pulsation theory, and stellar evolution theory has led both to changes in interpretations of observational results (which have been mistaken for observational results themselves) and to changes in the assumptions that must be made in order to produce concrete theoretical calculations. Thus, analysis of the pulsational characteristics of classical pulsators has led to improved interpretations of the observations and to a better understanding of the internal structure of evolving stars.

As a first example, consider those stars in galactic globular clusters that are burning helium in their cores while on the horizontal branch. Those stars that are at the same time in the instability strip which intersects this branch are known as RR Lyrae stars. They fall into two groups: those with short period and symmetric light curves known as $c$-type variables and those with longer periods and asymmetric light curves known as $a b$-type variables. The $c$-type variables occupy a region in the left hand or hotter portion of the instability strip and the $a b$-type variables occupy the right hand portion.

How does one know that variables in the shorter period group are oscillating in the first overtone while those in the long period group are oscillating in the fundamental mode? Using pulsation theory and model stellar envelopes one can construct a relationship between period $P_{F}$ in the fundamental radial mode, the radius of the envelope, and the assumed mass of the underlying stellar interior. This relationship is roughly (see, e.g., Böhm-Vitense et al., 1975)

$$
P_{F}^{2} \sim 0.00716\left(R^{3} / M\right)(R / M)^{0.445},
$$

and it is to a very good approximation independent of the composition. The period $P_{H}$ of the first overtone or first 'harmonic' is related to $P_{F}$ by $\log P_{H} \simeq \log P_{F}-0.13$. From the photometric data, as filtered through a stellar atmosphere analysis, one may determine the relative radii of stars in the instability strip and determine $\mathrm{d} P / \mathrm{d} R$ for the observed stars. One finds that $(\mathrm{d} P / \mathrm{d} R)_{\text {obs }}$ agrees with the theoretical result with $M \sim$ constant and, if 0.13 is added to the log of the period of all of the $c$-type variables, they fall on a continuum in the $\log P-\log R$ plane with the $a b$-type variables. It follows, of course, that we have evidence that the RR Lyrae variables are radial pulsators with the $c$-type variables oscillating in the first overtone and the $a b$ variables oscillating in the fundamental mode. The clincher comes when one constructs theoretical full amplitude nonlinear light curves showing the symmetry properties consistent with the observations and with the classification into fundamental and first overtone oscillators 
obtained simply by using results of linear pulsation analysis (see, e.g., Christy, 1966).

So far, we have learned nothing about stellar structure and evolution. This is achieved by estimating RR Lyrae masses with the help of Equation (1) and an assumption about the absolute luminosity of RR Lyrae stars (needed to estimate $R$ absolutely). This may be done in one of two ways. Either one resorts to main sequence fitting (dangerous because the location of theoretical Main Sequences are composition dependent and because the globular cluster heavy element abundances $Z$ are an order of magnitude smaller than the $Z$ appropriate to comparison clusters whose distances are known by parallax) or one uses the predictions of stellar evolution theory (dangerous because these predictions are functions of assumed $Z$ and $Y$ ).

In any case, making use of either scheme, one finds that $\log L_{H B} \simeq 1.6 \pm 0.1$ (e.g. Sweigart and Gross, 1976; Sandage et al., 1981). Using this luminosity in conjunction with an observed surface temperature one may find $R$ for any given RR Lyrae star. Inserting this $R$ and an observed period into Equation(1) then gives the mass $M=M_{R R} \simeq 0.6 M_{\odot}$. This is substantially less than the mass $M \simeq 0.8 M_{\odot}$ of a star near the Main Sequence turnoff in a globular cluster. Thus one discovers that, somewhere in the interim between the core hydrogen burning phase (the Main Sequence) and the core helium burning phase (the horizontal branch), a low mass star loses a substantial fraction of its original mass. For a popular discussion of this result, see Iben (1970, 1971).

A comparison between linear non adiabatic pulsation theory and the observations can give further information about the bulk properties of RR Lyrae stars and their progenitors. Non-adiabatic pulsation theory provides a composition-dependent estimate of the location of the blue edge of the instability strip for pulsation in any given radial mode. For horizontal branch stars the relevant mode is the first harmonic. One finds (Tuggle and Iben, 1973)

$$
(0.2-Y)+4.9\left(\log T_{H B E}-3.859\right)+0.35\left(\log P_{H B E}+0.55\right) \simeq 0,
$$

where $Y$ is the abundance by mass of helium, $T_{H B E}$ is the surface temperature at the blue edge of the distribution of $c$-type variables, and $P_{H B E}$ is the mean period of stars near this edge. Expression (2) is, to first order, independent of $Z$. Adopting $\log T_{H B E} \simeq 3.863 \pm 0.01$ (probably too large an error estimate by a factor of 2 ) and $\log P_{H B E} \simeq-0.55 \pm 0.05$, one obtains $Y \simeq 0.22 \pm 0.07( \pm 0.05$ is perhaps more reasonable).

Thus, with the help of the observational and theoretical properties of RR Lyrae stars, we have been able not only to establish the existence of a mass loss process (which theory cannot as yet produce from first principles) but have been able to show that the abundance of helium in the odest stars is substantially greater than zero. This latter result provides support for the inference from the characteristics of the $3 \mathrm{~K}$ background radiation that nucleosynthesis in the early Universe produced a helium abundance of about $23 \%$ by mass.

As a next example, consider the classical Cepheids which have been used in establishing the second step in the extragalactic distance scale. These are Cepheids in 
galactic clusters whose absolute distances and luminosities can be estimated by fitting the cluster Main Sequence to an extrapolation of the Hyades main sequence (the traditional first step in the distance scale).

Because they are pulsating, one may obtain an estimate of the mass of each Cepheid with the help of the results of pulsation theory. For stars with characteristics in the ranges $\left(2<M / M_{\odot}<10,2.75<\log L<4.75,3.65<\log T_{e}<3.85\right)$, linear non-adiabatic pulsation theory gives (Iben and Tuggle, 1975)

$$
\begin{aligned}
\log P_{F} \simeq & 0.71-3.34\left(\log T_{e}-3.75\right)+0.85(\log L-3.25)- \\
& -0.62(\log M-0.7)
\end{aligned}
$$

or

$$
P_{F} \simeq \text { constant }\left(R^{3} / M\right)^{1 / 2} R^{0.2} / M^{0.12}
$$

again roughly independent of composition. Here $P_{F}$ is the pulsation period in days, $T_{e}$ is the surface temperature in Kelvins, and $M, L$, and $R$ are stellar mass, luminosity, and radius, all in solar units. Clearly, if $P, T_{e}$, and $L$ are known, $M$ may be determined from Equation (3). The mass $M=M_{\text {pulse }}$ estimated in this way may then be compared with the mass $M=M_{\text {evo }}$ of a model intermediatemass star which evolves through the classical instability strip on a long-time scale during its core helium burning phase. By comparing theoretical evolutionary tracks with the location of the theoretical instability strip, one finds the following relationship between $M_{\text {evo }}, L$, and composition of a model crossing the strip (Becker et al., 1977 = BIT):

$$
\log L \simeq(0.46-41 \Delta Z+6.6 \Delta Y)+(3.68+21 \Delta Z-4.5 \Delta Y) \log M_{\mathrm{evo}},
$$

where $\Delta Z=Z-0.02$, and $\Delta Y=Y-0.28$. For the 13 Cepheids in question, $\Delta Z=\Delta Y=0$ are reasonable choices, and insertion into Equation (4) of $L$ obtained by Main Sequence fitting gives a first estimate of $M_{\text {evo }}$. When luminosities estimated by adopting a distance modulus of $\sim 3.05 \mathrm{mag}$. for the Hyades are used, one finds that $M_{\text {pulse }}$ is typically $20-40 \%$ smaller than $M_{\text {evo }}$ (Cogan, 1970; Rogers, 1970; Iben and Tuggle, 1972) and one might infer once again that evidence for considerable mass loss during the interim between the Main Sequence and the core helium burning phases has been uncovered. However, in this case, the lifetime of the red giant phase which precedes the Cepheid phase (and which is the most likely place for extensive mass loss to occur) is so short that one would require mass loss rates on the order of several times $10^{-5} M_{\odot} \mathrm{yr}^{-1}$ to explain a $30 \%$ decrease in mass (a typical Cepheid mass is $3-6 M_{\odot}$ ) and such high rates for normal intermediate-mass red giants (of luminosity $\log L \sim 3-4$ ) are not found observationally.

Inspection of Equations (3) and (4) shows that a modest increase of about 0.25 mag. in the assumed distance modulus to the Hyades will remove the discrepancy between $M_{\text {pulse }}$ and $M_{\text {evo }}$ (Iben and Tuggle, 1972). In the last decade, a number of investigations have shown that such an increase is not inconsistent with the data used to derive the Hyades distance (parallax measurements, convergent point assumption, etc.). References to the relevant literature are given by Cox (1980). 
Thus, pulsation theory and evolution theory have participated in the establishment of a reliable measure of the traditional first step in the distance scale. They may participate also in the establishment of the second step, namely the distance to Andromeda. In addition to producing Equation (3), linear non-adiabatic pulsation theory can provide a relationship between $T_{e}, L, M, P$, and composition along the blue edge for pulsation in the fundamental mode (relevant for the brightest Cepheids). It is clear that from this relationship and Equations (3) and (4) one may eliminate three unknowns to obtain a relationship between $L, P$, and composition. In practice it is more reliable (and easier) to draw theoretical blue edges in the $\mathrm{H}-\mathrm{R}$ diagram for each choice of $M$ and composition, adopt a width of, say, $\Delta \log T_{e} \simeq 0.04$ for the instability strip, and find where the evolutionary track for a model of the chosen mass and composition crosses the semiempirical instability strip. In this way one finds that (BIT)

$$
\langle\log L\rangle \simeq 2.51+1.25\langle\log P\rangle
$$

where $\langle\log L\rangle$ is the average luminosity in the strip and $\langle\log P\rangle$ is the average period.

A remarkable feature of relationship (5) is that it holds reasonably well for all compositions, thus providing at long last theoretical justification for the observers assumption that the period-luminosity relationship is independent of composition. Note further that the theoretical relationship is indeed that: only through the adoption of a width for the instability strip have results of observations entered (weakly) into establishing the relation. I believe that this is a triumph of both stellar evolution and pulsation theory of a magnitude that has yet to be appreciated.

In any case, using Equation (5) to establish the distance to Andromeda requires observational estimates of $P$ and apparent bolometric magnitude for a number of Cepheids, and the equivalent of (5) that has been derived by normalizing to the 13 galactic Cepheids whose distances can be estimated by Main Sequence fitting is (when corrected by 0.25 mag.!) just as good a relationship as (5). Given this, perhaps the best conclusion to draw is that the remarkable similarity between the theoretical $P-L$ relationship and the observationally based relationship tells us that our interior models of core helium burning stars of intermediate mass are perhaps believable.

As a final example, consider stars in the asymptotic giant branch (AGB) phase of evolution. These are stars with dense carbon-oxygen cores in which electrons are highly degenerate. Nuclear burning occurs alternatively in two shells outside of this core. Most of the time hydrogen-burning in the outermost shell provides the luminosity escaping from the surface. However, at regular intervals, helium-burning in the innermost shell becomes unstable and the star experiences a thermal pulse (Schwarzschild and Härm, 1965; Weigert, 1965). The time between pulses varies from $\sim 10^{5} \mathrm{yr}$ when core mass $M_{c}=0.6 M_{\odot}$ to $\sim 10^{3} \mathrm{yr}$ when $M_{c}=1 M_{\odot}$ and the quiescent helium burning phase which follows each pulse last approximately one fourth as long as the quiescent helium burning phase. The pulse itself lasts from $300 \mathrm{yr}\left(M_{c}=0.6 M_{\odot}\right)$ to $30 \mathrm{yr}\left(M_{c}=1 M_{\odot}\right)$.

There exists a simple relationship between the maximum luminosity of an AGB star during the quiescent hydrogen-burning phase and the core mass. In good approximation 
(Paczynski, 1971; Uus, 1971)

$$
L=6 \times 10^{4} L_{\odot}\left(M_{c} / M_{\odot}-0.5\right) .
$$

From this relationship (with the knowledge that $M_{c}^{\min } \simeq 0.53 M_{\odot}$ and $M_{c}^{\max }=1.4 M_{\odot}$ ) one sees that AGB stars occur typically at magnitudes in the range

$$
-7.3<M_{\mathrm{BOL}}<-3.3 \text {. }
$$

AGB stars spend most of their lives far to the red of the classical instability strip and they possess extremely deep convective envelopes. However, because of the low density in the envelopes, convection is not efficient and the temperature gradient is highly superadiabatic. Driving occurs in the hydrogen ionization zone and the stars oscillate as Miras (low luminosity) or as long period variables (high luminosity). Unfortunately, convection once again interferes with understanding. Because the degree of superadiabaticity is large over much of the envelope and is quite sensitive to the treatment of convection (this time, the time dependence is also important), a unique PLT $_{e} \mathbf{M}$ relationship does not exist for any mode of oscillation. Furthermore, results depend also on uncertain molecular opacities and on surface boundary conditions (Fox and Wood, 1981). Hence, not only can one not use pulsation theory to estimate mass, as in the case of RR Lyrae stars and Cepheids, there is even controversy over the mode in which an AGB star oscillates (see, e.g., Willson, 1981; Wood, 1981).

Given the difficulty of ascertaining directly the global chacteristics of AGB stars by using observed pulsation properties in conjunction with an adequate pulsation theory, it is encouraging that there exists an indirect way of using pulsation theory to learn something interesting about AGB stars. This way relies on the fact that every Cepheid in the period range 2-10 days should ultimately turn into an AGB star of high luminosity. That this is true is illustrated in Table II, which gives various properties of 12 Cepheids used in the classic derivation of the distance scale. Relevant observational data used

\section{TABLE II}

Properties of 12 classical Cepheids in galactic clusters

\begin{tabular}{lccccccc}
\hline Star & $M_{\text {BOL }}$ & $\log M$ & $\log P$ & Age & $M$ & $M_{\text {CO }}^{\min }$ & $M_{\text {BOL }}^{\text {AGB }}$ \\
\hline EV Sct & -2.90 & 0.615 & 0.489 & 11.7 & 4.12 & 0.74 & -5.6 \\
CE Casb & -3.49 & 0.676 & 0.651 & 8.8 & 4.74 & 0.82 & -5.9 \\
CECas & -3.39 & 0.658 & 0.687 & 8.2 & 4.55 & 0.79 & -5.8 \\
CECasa & -3.58 & 0.710 & 0.710 & 7.9 & 5.13 & 0.84 & -6.0 \\
UY Per & -3.83 & 0.738 & 0.729 & 7.6 & 5.47 & 0.86 & -6.2 \\
VYPer & -4.19 & $(\sim 0.70)$ & $(0.742)$ & $(<7.4)$ & $\sim 5$ & 0.83 & -6.0 \\
USgr & -4.21 & 0.758 & 0.828 & 6.4 & 5.73 & 0.88 & -6.1 \\
DLCas & -4.17 & 0.754 & 0.903 & 5.6 & 5.68 & 0.88 & -6.1 \\
SNor & -4.37 & 0.757 & 0.989 & 4.8 & 5.71 & 0.88 & -6.1 \\
VX Per & -4.63 & 0.748 & 1.037 & 4.4 & 5.62 & 0.87 & -6.1 \\
SZ Cas & -5.01 & 0.781 & 1.134 & 3.7 & 6.04 & 0.90 & -6.2 \\
RS Pup & -6.31 & 1.019 & 1.616 & 1.6 & 10.4 & & supergiant
\end{tabular}


to prepare this table are from Sandage and Tammann (1969), Sandage and Gratton (1963), and Rogers (1970). The bolometric magnitudes are $0.25 \mathrm{mag}$. brighter and the logarithms of the masses are 0.1 larger than given in Table II of Iben and Tuggle (1972). Cepheid ages (in $10^{7} \mathrm{yr}$ ) are estimated using Equation (20) in BIT. Of primary interest here are the core mass $M_{\mathrm{CO}}^{\mathrm{min}}$ and the bolometric magnitude $M_{\mathrm{BOL}}^{\mathrm{AGB}}$ which each Cepheid should ultimately attain when it first transforms into an AGB star and begins to pulse thermally. The relationship between initial mass $M$ and $M_{\mathrm{CO}}^{\min }$ is taken from Iben (1981) and the bolometric magnitude follows from Equation (6).

Since the theoretical lifetime of a thermally pulsing (TP) AGB star and the lifetime of a typical Cepheid are roughly the same $\left(\sim 10^{6} \mathrm{yr}\right)$, one expects to find approximately a dozen TP-AGB stars in the galactic clusters containing the studied Cepheids. As Table II shows, many of these AGB stars should be brighter than $M_{\mathrm{BOL}}=-6$.

Theory also predicts that TP-AGB stars with cores as large as those in Table II should be dredging up carbon to their surfaces (see, e.g., Iben, 1981, for a recent discussion) and that roughly half of their AGB lifetime should be spent as carbon stars (abundance of carbon greater that the abundance of oxygen at the surface). Significantly, not one of the galactic clusters chosen for study contains a carbon star, whereas there should be altogether about 6 .

This point is made even more graphically by the bright stars in the cluster NGC 1866 in the large Magellanic Cloud. This cluster contains 7 Cepheids and at least four $\mathrm{AGB}$ stars ranging in brightness from $M_{\mathrm{BOL}}=-5$ to $M_{\mathrm{BOL}} \simeq-6.2$ (Frogel and Blanco, 1981). Using pulsation theory and the relevant observed properties of the Cepheids one finds that the average Cepheid mass is $M_{\mathrm{Ceph}} \sim 4-5 M_{\odot}$ and $M_{\mathrm{BOL}}^{\mathrm{AGB}} \cong-5.6$ to $-6.1 \mathrm{mag}$. Not one of the AGB stars is a carbon star.

There are at least two possible interpretations of these results. Either evolution theory (which says that about half of the bright TP-AGB stars should be carbon stars) is wrong; or, when it becomes a carbon star, the lifetime of a bright AGB star is short compared to its lifetime as a non-carbon star. The existence of a class of carbon stars in our own Galaxy with estimated mass loss rates greater than $10^{-5} M_{\odot} \mathrm{yr}^{-1}$ (Knapp et al., 1982) suggests that the second possibility is the more likely one. The inference is that, when $\mathrm{C}$ exceeds $\mathrm{O}$ at the stellar surface, a 'superwind' is engendered (perhaps due to radiation pressure on carbon grains).

To summarize, the fact that, in those clusters containing Cepheids, the brightest AGB stars are not carbon stars is support for the theoretical prediction that bright AGB stars will become carbon stars! Since theoretically $\mathrm{C}$ exceeds $\mathrm{O}$ at the surface well before the core mass reaches the Chandrasekhar limit of about $1.4 M_{\odot}$, we have also learned that single stars of intermediate mass do not become supernovae! These are rather sweeping conclusions, and they have been made possible with the help of a seismological analysis of classical variables.

May the helioseismologists ultimately be as successful! 


\section{References}

Becker, S. A., Iben, I. Jr., and Tuggle, R. S.: 1977, Astrophys. J. 218, 633.

Bohm-Vitense, E., Szkody, P., Wallerstein, G., and Iben, I. Jr.: 1974, Astrophys. J. 194, 125.

Christy, R.: 1966, Astrophys. J. 144, 108; 145, 337.

Cogan, B. C.: 1970, Astrophys. J. 162, 129.

Cox, J. P.: 1974, Rep. Prog. Phys. 37, 563.

Cox, A. N.: 1980, Ann. Rev. Astron. Astrophys. 18, 15.

Davis, R.: 1981, private communication.

Fox, M. W. and Wood, P. R.: 1981, preprint.

Frogel, J. A. and Blanco, V. M.: 1982, private communication.

Iben, I. Jr.: 1970, Scientific American, July issue.

Iben, I. Jr.: 1971, Publ. Astron. Soc, Pacific 83, 697.

Iben, I. Jr.: 1975, Astrophys. J. 197, 39.

Iben, I. Jr.: 1981, Astrophys. J. 246, 278.

Iben, I. Jr. and Mahaffy, J.: 1976, Astrophys. J. 209, L39.

Iben, I. Jr. and Tuggle, R. S.: 1972, Astrophys. J. 178, 433.

Knapp, G. R., Phillips, T. G., Leighton, R. B., Lo, K. Y., Wannier, P. G., Wooten, H. A., and Huggins, P. J.: 1982, Astrophys. J. 252, 616.

Paczynski, B.: 1970, Acta. Astron. 20, 47, 287.

Rogers, A. W.: 1970, Monthy Notices Roy. Astron. Soc. 151, 133.

Sandage, A. R. and Gratton, L.: 1963, Star Evolution, Academic Press, New York, p. 11.

Sandage, A. R. and Tammann, G. A.: 1969, Astrophys. J. 157, 683.

Sandage, A., Katem, B., and Sandage, M.: 1981, Astrophys. J. 46, 41.

Schwarzschild, M. and Härm, R.: 1965, Astrophys. J. 142, 855.

. Severny, A. B., Kotov, V. A., and Tsap, T. T.: 1976, Nature 259, 87.

Sweigart, A. V. and Gross, P. G.: 1976, Astrophys. J. 32, 367.

Sweigart, A. V. and Gross, P. G.: 1978, Astrophys. J. 36, 405.

Tuggle, R. S. and Iben, I. Jr.: 1973, Astrophys. J. 186, 593.

Uus, U.: 1970, Nauch. Inform. Acad. Nauk USSR 17, 3, 25, 35, 48.

Weigert, A.: 1966, Z. Astrophys. J. 64, 395.

Willson, L. A.: 1981, in I. Iben Jr. and A. Renzini (eds.), Physical Pracesses in Red Giants, D. Reidel Publ. Co., Dordrecht, Holland, p. 225.

Wood, P. R.: 1981, in I. Iben Jr, and A. Renzini (eds.), Physical Processes in Red Giants, D. Reidel Publ. Co., Dordrecht, Holland, p. 205. 